\title{
THE PROPAGATION OF A PLANE SHOCK INTO A QUIET ATMOSPHERE II
}

\author{
M. H. MARTIN
}

1. Introduction. In a previous paper (3) the study of one-dimensional, unsteady flows, isentropic or anisentropic, was reduced to the integration of a Monge-Ampère partial differential equation

$$
\xi_{\psi \psi} \xi_{p p}-\xi_{\psi p}^{2}=\tau_{p}
$$

For a polytropic gas, the specific volume

$$
\tau=e^{\left(S-S_{0}\right) / c_{p}} \cdot p^{-n}
$$$$
n=1 / \gamma,
$$

takes the form

$$
\tau=\delta(\psi) p^{-n}
$$

once the entropy distribution function $S=S(\psi)$ is specified. A solution $\xi=\xi(\psi, p)$ having been determined by one means or another, the actual flow is presented by the mapping

$$
x=\int\left\{\left(\xi_{\psi \psi} \xi_{\psi p}+\tau\right) d \psi+\xi_{\psi} \xi_{p p} d p\right\}, \quad t=\xi_{p}, \quad\left(u=\xi_{\psi}\right),
$$

of the $(\psi, p)$-plane upon the $(x, t)$-plane. This mapping carries the rectilinear network $\psi=$ const., $p=$ const., in the $(\psi, p)$-plane into the curvilinear network of trajectories and isobars in the $(x, t)$-plane.

A progressive condensation shock, carrying in back of it the values $u, \tau, p$ of the velocity, specific volume and pressure and moving into a quiet atmosphere where these quantities have fixed values $u_{0}, \tau_{0}, p_{0}$ is governed by the shock conditions:

$$
\begin{aligned}
u & =u_{0}+\sqrt{ }\left(p-p_{0}\right)\left(\tau_{0}-\tau\right), \\
\frac{d x}{d t} & =u_{0}+\tau_{0} \sqrt{\frac{p-p_{0}}{\tau_{0}-\tau}} \\
\tau & =\tau_{0} \frac{(\gamma-1) p+(\gamma+1) p_{0}}{(\gamma+1) p+(\gamma-1) p_{0}}
\end{aligned}
$$

where (4.2) gives the shock velocity.

Once the entropy distribution function $S(\psi)$ is selected, the determination of the motion of the shock into the quiet atmosphere and of the flow immediately behind it, sets (3) a Problem of Cauchy for the controlling partial differential equation (1).

Received August 28, 1954. This work was carried out under Contract DA-36-034-ORD-1486 with the Office of Ordnance Research, U.S. Army. 
If, however, one is willing to forgo knowledge of the flow immediately in back of the shock, the motion of the shock into the quiet atmosphere can be determined without solving the Problem of Cauchy. In this paper we investigate the influence of the choice of the entropy distribution function $S(\psi)$ upon the propagation of the shock into the quiet atmosphere. Among other things we find that the time required (2, p. 213) for complete decay of the shock may be finite or infinite, depending upon the selection of the entropy distribution function.

2. The shock curve. If we substitute from (2) for $\tau$ in (4.3) and prescribe an entropy distribution function $S(\psi)$, the resulting equation

$$
S(\psi)-S_{0}=c_{p} \log \left(\tau_{0} \frac{(\gamma-1) p+(\gamma+1) p_{0}}{(\gamma+1) p+(\gamma-1) p_{0}} p^{n}\right)
$$

defines a curve $\psi=\psi(p)$ in the $(\psi, p)$-plane. This curve transforms by (3) into the shock curve in the $(x, t)$-plane and we propose to deduce its parametric equations

$$
x=x(p), \quad t=t(p),
$$

for a given entropy distribution function $S(\psi)$. Here $p$ denotes the pressure immediately in back of the shock.

From (3) we see that a curve $\psi=\psi(p)$ in the $(\psi, p)$-plane is carried into a curve $x=x(t)$ in the $(x, t)$-plane along which

$$
\frac{d x}{d t}=u+\tau \frac{d \psi}{d p} / \frac{d t}{d p} .
$$

As a matter of fact this result is an immediate consequence of the relation

$$
d \psi=\rho d x-\rho u d t,
$$

i.e., of the principle of conservation of mass.

We take (5) for the curve $\psi=\psi(p)$, substitute in (7) for $u$ and $d x / d t$ from (4.1) and (4.2), to obtain

$$
\frac{d t}{d p}=\frac{d \psi}{d p} \sqrt{ }\left(\frac{\tau_{0}-\tau}{p-p_{0}}\right) .
$$

When $\tau$ is eliminated from this by (4.3) we find

$$
\left.t=\int \frac{d \psi}{d p} \sqrt{\left(\frac{2 \tau_{0}}{(\gamma+1) p+(\gamma-1) p_{0}}\right)}\right) d p,
$$

to reduce the determination of the function $t(p)$ in (6) to a quadrature, once the function $\psi=\psi(p)$ is fixed by the selection of $S(\psi)$ in (5).

To determine the function $x(p)$ in (6), we write (4.2) in the form

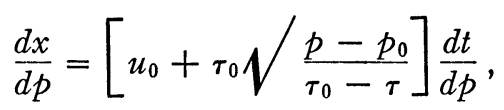

and substitute in here for $d t / d p$ from (9). This yields 


$$
x=\tau_{0} \psi+u_{0} t
$$

where $\psi, t$ are the functions of $p$ defined in (5) and (10). This result may be checked by applying the principle of conservation of mass across the shock in the form

$$
d \psi=\rho d x-\rho u d t=\rho_{0} d x-\rho_{0} u_{0} d t .
$$

We sum up our results on the shock curve in the theorem below.

THEOREM. For a shock moving into a quiet atmosphere in which the velocity $u$, specific volume $\tau$, and pressure $p$ have fixed values $u_{0}, \tau_{0}, p_{0}$, the shock curve in the $(x, t)$-plane is described parametrically in terms of the pressure $p$ immediately in back of the shock by

$$
\left.x=\tau_{0} \psi+u_{0} t, t=\int \frac{d \psi}{d p} \sqrt{\left(\frac{2 \tau_{0}}{(\gamma+1) p+(\gamma-1) p_{0}}\right)}\right) d p,
$$

in which the function $\psi(p)$ is defined implicitly by

$$
S(\psi)=c_{p} \log \left(\tau_{0} \frac{(\gamma-1) p+(\gamma+1) p_{0}}{(\gamma+1) p+(\gamma-1) p_{0}} p^{n}\right)+S_{0}, \quad S_{0}=\text { const., }
$$

upon prescription of the entropy distribution function $S(\psi)$.

3. Shock decay. The problem of shock decay is of some interest $(\mathbf{1} ; \mathbf{2})$ and we shall make some remarks on how decay is affected by the choice of the entropy distribution function.

First of all, for a shock to decay completely it is necessary and sufficient that the pressure $p$ in back of the shock equal the pressure $p_{0}$ in front of the shock.

Starting with a pressure $p_{1}>p_{0}$ in back of the shock, it is clear from the above theorem that complete decay will require a finite or an infinite time, according as the integral

$$
\left.t\left(p_{0}\right)-t\left(p_{1}\right)=\int_{p_{1}}^{p_{0}} \frac{d \psi}{d p} \sqrt{\left(\frac{2 \tau_{0}}{(\gamma+1) p+(\gamma-1) p_{0}}\right)}\right) d p
$$

converges or diverges.

To fix the ideas, let us take $u_{0}=0$, so that the gas in front of the shock is at rest, and assume that the entropy distribution function $S(\psi)$ is chosen so that

$$
-\infty<\frac{d \psi}{d p}<0 \text { for } p_{1} \leqslant p<p_{0}
$$

From the parametric equations (12) of the shock curve it is clear that $x$ and $t$ both increase monotonically for decreasing $p$ as shown in Fig. 1.

If the entropy distribution function $S(\psi)$ is taken so that $d \psi / d p$ is finite at $p_{0}$, or is infinite for $p=p_{0}$ to an order low enough so that the integral (13) still converges, the shock curve will end at a finite point $P_{0}$ in the $(x, t)$-plane. Under these conditions the shock decays after covering a finite distance in a finite time. 


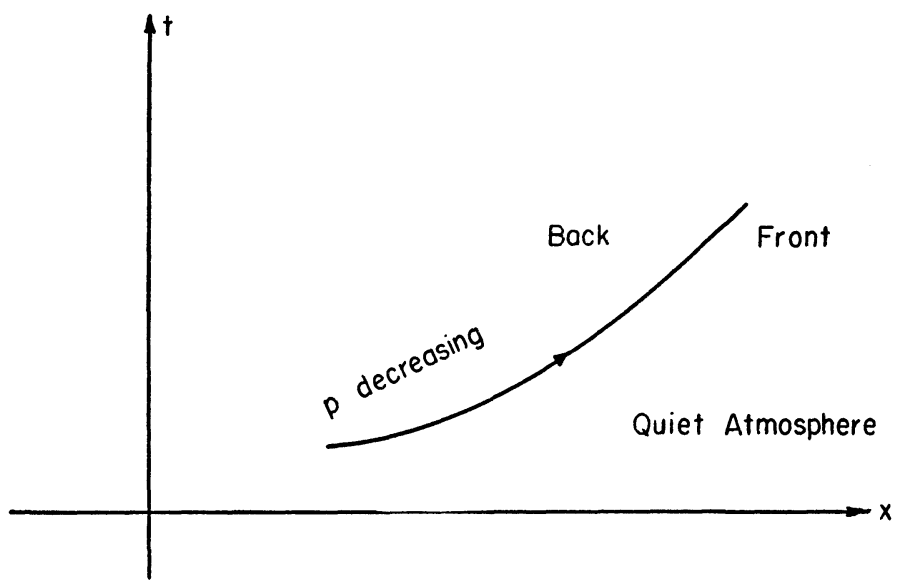

FIGURE 1. The shock curve for $d \psi / d p<0$.

On the other hand, if $S(\psi)$ be chosen so that $d \psi / d p$ is infinite for $p=p_{0}$ and the integral (13) diverges, both $x$ and $t$ become infinite as $p$ decreases to $p_{0}$. In this case the shock travels an infinite distance and requires an infinite time before it decays.

Decay after travelling an infinite distance in a finite time or a finite distance in an infinite time is accordingly not possible.

Let us write (5) in the form

$$
S(\psi)-S_{0}=c_{p} \log \left[\frac{(\gamma-1) p+(\gamma+1) p_{0}}{(\gamma+1) p+(\gamma-1) p_{0}}\left(\frac{p}{p_{0}}\right)^{n}\right], \quad S_{0}=\text { const. }
$$

in which $S_{0}$ may be interpreted as the specific entropy of the quiet atmosphere in front of the shock. Expansion of the second member in powers of $p-p_{0}$ yields

$$
S(\psi)-S_{0}=A\left(p-p_{0}\right)^{3}+\ldots, \quad A=n\left(1-n^{2}\right) c_{p} / 12 p_{0}^{3},
$$

and differentiation gives

$$
S^{\prime}(\psi) \frac{d \psi}{d p}=c_{p}\left(\gamma-\gamma^{-1}\right) \frac{\left(p-p_{0}\right)^{2}}{p\left[(\gamma-1) p+(\gamma+1) p_{0}\right]\left[(\gamma+1) p+(\gamma-1) p_{0}\right]},
$$

where $S^{\prime}(\psi)$ is the derivative of $S(\psi)$.

From (17) it is clear that (14) requires $S^{\prime}<0$, i.e., that the entropy distribution function be monotone decreasing. Furthermore the shock will require a finite time to decay if $S^{\prime}(\psi)$, expressed as a function of $p$, vanishes to less than the third order in $p-p_{0}$; on the other hand, if $S^{\prime}(\psi)$ vanishes to the third or a higher order in $p-p_{0}$, the time for decay is infinite.

4. Examples of shock decay. We illustrate the general principles in the previous section by simple examples. 
For the first example, take

$$
S(\psi)=S_{0}-\psi,
$$

so that, from (16)

$$
\psi=-A\left(p-p_{0}\right)^{3}+\ldots, \quad \frac{d \psi}{d p}=-3 A\left(p-p_{0}\right)^{2}+\ldots
$$

from which and (13) it is clear that the shock decays in finite time.

For the second example, take

$$
S(\psi)=S_{0}+\psi^{-1},
$$

for which

$$
\psi=A^{-1}\left(p-p_{0}\right)^{-3}+\ldots, \frac{d \psi}{d p}=-3 A^{-1}\left(p-p_{0}\right)^{-4}+\ldots,
$$

and it is clear again from (13) that shock decay now requires an infinite time.

More generally let us take

$$
S(\psi)=S_{0}-\frac{\psi^{k}}{k}, \quad k= \pm 1, \pm 3, \ldots
$$

Clearly $S^{\prime}(\psi)<0$ for all stated $k$, and its expansion in powers of $p-p_{0}$ is

$$
S^{\prime}(\psi)=-B\left(p-p_{0}\right)^{3(1-1 / k)}+\ldots,
$$

where $B$ is a positive constant. Thus $S^{\prime}(\psi)$ will vanish to an order less than three at $p_{0}$ for $k=1,3, \ldots$ and the shock decays in finite time; for $k=-1$, $-3, \ldots S^{\prime}(\psi)$ vanishes to a higher order than 3 at $p_{0}$ and decay requires an infinite time.

\section{REFERENCES}

1. S. Chandrasekar, On the decay of plane shock waves, Aberdeen Proving Ground, BRL Report No. 423 (1943).

2. K. O. Friedrichs, Formation and decay of shock waves, Comm. on Appl. Math., 1 (1948), 211-245.

3. M. H. Martin, The propagation of a plane shock into a quiet atmosphere, Can. J. Math., 5 (1953), 37-39.

Institute for Fluid Dynamics and Applied Mathematics

University of Maryland 